\title{
Gestión en Línea de las Evidencias de Aprendizaje en Educación Superior
}

\author{
Cornelio Cano y Sara C. Hernández \\ Departamento de Sistemas de Información, Centro Universitario de Ciencias Económico \\ Administrativas, Universidad de Guadalajara, Periférico Sur 799, 45100. Zapopan, Jalisco.- \\ México (e-mail: ccano@cucea.udg.mx, shernand@cencar.udg.mx )
}

Recibido May. 18, 2011; Aceptado Jul. 20, 2011; Versión final recibida Ago. 17, 2011

\begin{abstract}
Resumen
El propósito de esta investigación fue verificar la percepción de estudiantes universitarios sobre la integración de la tecnología informática en los procesos de enseñanza-aprendizaje. Se utilizó el método de investigación-acción para diseñar, implementar y poner en práctica un modelo para gestionar en línea evidencias de aprendizaje, con el uso de herramientas web de libre acceso. Para verificar la pertinencia y funcionalidad del modelo, se aplicó una encuesta entre los estudiantes que lo utilizaron en un primer curso universitario de Estadística, para la preparación, conservación y presentación de los productos de tareas realizadas fuera de clase. Los resultados muestran que los estudiantes perciben la necesidad de integrar la tecnología informática en la educación superior. También ven algunos beneficios en el aprendizaje con el uso de esta tecnología.
\end{abstract}

Palabras clave: evidencias de aprendizaje, investigación-acción, herramientas web, entornos mixtos

\section{Online Management of Learning Evidence in Higher Education}

\begin{abstract}
The purpose of this research was to verify the perception of university students on the integration of computer technology in teaching and learning processes. The action research method was used to design, deploy and implement a model for online managing of learning evidence using free available web tools. To verify the relevance and functionality of the model, a survey was done among students who used it in a first university course of statistics, for creating, saving and presenting their homework's products. The results show that students perceive the need to integrate information technology in higher-education. They also see some learning benefits with the use of this technology.
\end{abstract}

Keywords: learning evidence, action-research, web tools, b-learning 


\section{INTRODUCCIÓN}

La participación de la tecnología informática en la educación superior es un aspecto que adquiere cada vez más importancia; la disponibilidad de herramientas computacionales que ofrecen la posibilidad de simplificar algunas actividades docentes produce un interés creciente en profesores que buscan, a través de esta tecnología, mejorar la efectividad en su práctica docente. "La era digital trae consigo cambios profundos. Estos cambios pueden ser benéficos para la humanidad, si somos capaces de planear adecuadamente sus consecuencias" (Palfrey y Gasser, 2008). En la educación superior, la integración de esta tecnología presenta diversos retos a la actividad docente (Ginns y Ellis, 2007); debido a la dinámica misma de su evolución, las soluciones que se desarrollan con base en esta tecnología se revisan con frecuencia, a fin de prevenir la obsolescencia, dado que en ocasiones se observa que durante el tiempo en que se realizan esfuerzos para adoptar una aplicación en particular, aparecen otras que ofrecen más posibilidades de remplazarla con ventaja (Laurillard, 2006).

La situación anterior representa una carga de trabajo adicional para los docentes, quienes no siempre perciben con claridad los posibles beneficios de la tecnología computacional en el aula; Según Bates (2001) la tecnología debe utilizarse para subsanar deficiencias del sistema tradicional actual de enseñanza o para aportar algunas ventajas, aún cuando, en ocasiones, sus beneficios son difíciles de cuantificar. La capacidad de los sistemas informáticos para almacenar, procesar y transmitir información de manera efectiva permite considerar su utilización en actividades donde se requiere de esta capacidad para lograr mayor efectividad en su ejecución, como es el caso de la gestión de documentos que se producen como evidencias del aprendizaje alcanzado; el avance en el logro de los objetivos de instrucción se expone mediante la publicación de evidencias que documentan el nivel de progreso alcanzado (Barberá et al., 2006). Este es el aspecto que se aborda en el presente artículo, donde se describe un modelo basado en herramientas web de acceso libre, que permite simplificar el manejo de estas evidencias.

La evaluación del aprendizaje se refiere a los métodos que se aplican para determinar el nivel de conocimientos alcanzado por un estudiante en un proceso educativo (Gipps, 1994). Es parte fundamental en los procesos de enseñanza-aprendizaje. Los estudiantes tienden a definir sus estrategias de estudio de acuerdo a los métodos que utilizan los profesores para evaluar su desempeñon, más que en las técnicas y contenidos de la enseñanza (Norton, 2007); de ahí que una evaluación efectiva no sólo mide el desempeño del estudiante, también favorece el avance en el logro de los objetivos de instrucción. La diversidad de factores que intervienen en los procesos educativos ha propiciado la generación de diversos métodos para evaluar el aprendizaje; desde los tradicionales exámenes de opción múltiple, hasta los portafolios electrónicos que hacen uso de recursos informáticos cada vez más desarrollados (Barberá et al., 2006). Esta diversidad de métodos representa un reto al docente, que debe resolver al momento de decidir cómo realizar el proceso, "aún los profesores más experimentados revisan continuamente sus técnicas de evaluación para verificar que siguen siendo efectivas para medir el progreso y conocimiento de los estudiantes" (McDonald, 2011). La evaluación del aprendizaje consiste en algo más que asignar una calificación; además de medir el progreso del estudiante, muestra su potencial y limitaciones para avanzar en el conocimiento de la materia que se evalúa.

Debido a la importancia que conceden los estudiantes a la evaluación, es posible lograr resultados notables en su formación a través de ella, siempre que se le realice de manera adecuada. Marzano (2006) señala que una evaluación efectiva se resume en cuatro puntos: a) ser frecuente, b) de naturaleza formativa (propiciar la construcción del conocimiento), c) motivar al estudiante a superar su desempeño en forma constante y d) proporcionar una imagen clara de su progreso académico, con recomendaciones pertinentes para superarlo. De acuerdo a lo anterior, se puede afirmar que uno de los factores de más influencia en el avance académico de un estudiante es la realimentación adecuada y oportuna sobre su desempeño.

\section{FUNDAMENTOS TEÓRICOS}

La evaluación del aprendizaje puede ser de tres tipos: formativa, sumativa e ipsativa (Gipps, 1994). La evaluación formativa es la que se realiza durante el transcurso de la instrucción y tiene por 
objeto fortalecer al proceso de enseñanza-aprendizaje, a través de la comunicación eficaz y oportuna de los resultados a los estudiantes que se evalúan. La evaluación sumativa se refiere a la acumulación de los avances observados en el estudiante al final del proceso, o de alguna de sus etapas intermedias. Finalmente, la evaluación ipsativa, también llamada autoformativa, se refiere a la diferencia que percibe el estudiante entre sus conocimientos al inicio del proceso de instrucción y los que observa al finalizar el mismo. "La evaluación no sólo debe medir el aprendizaje logrado, sino también contribuir a su desarrollo" (James et al., 2002). El desarrollo de mejores prácticas de evaluación, con integridad y rigor académico, son cada vez más necesarias para mejorar la calidad de la educación superior.

Los aspectos que se consideran para caracterizar un método de evaluación son la validez y la confiabilidad. La validez se refiere al grado en que mide lo que se pretende medir; la fiabilidad, por su parte, indica la precisión y consistencia de los datos obtenidos en el proceso, refleja el grado de similitud en los resultados observados por dos personas al evaluar al mismo estudiante, o en dos evaluaciones realizadas al estudiante por una misma persona, en circunstancias similares (Gipps, 1994). Esta actividad permite determinar qué tan bien están aprendiendo los estudiantes, proporciona información a estudiantes, docentes, directivos y personas interesadas sobre los resultados de los procesos educativos y es parte integral de la búsqueda de mejores prácticas educativas. El proceso consiste en obtener la información pertinente, procesarla y formular juicios de valor sobre el nivel de conocimientos de la persona que se evalúa. Son procesos a través de los cuales los educadores utilizan las respuestas de los estudiantes a estímulos especialmente creados, o que ocurren de manera natural, para hacer inferencias sobre el conocimiento y habilidades de los estudiantes (Pellegrino et al., 2001).

Los recientes desarrollos tecnológicos están transformando las ideas sobre las competencias que los estudiantes deben desarrollar en su proceso educativo y las instituciones educativas tratan de responder a los cambios sociales, para redefinir qué es lo que deben aprender todos los estudiantes (Pellegrino et al., 2001). Estas tendencias tienen implicaciones profundas en la evaluación. Los cambios sociales, económicos y tecnológicos, principalmente, han modificado el concepto generalmente aceptado sobre el aprendizaje y las habilidades que las instituciones educativas deben desarrollar en sus estudiantes, así como los enfoques utilizados para evaluar estas habilidades.

Una práctica común para realizar la evaluación del aprendizaje es el de establecer desde el principio los aspectos que se toman en cuenta, con su nivel de ponderación o importancia. Se trata de un método sencillo y flexible que permite considerar todos los aspectos que el profesor considere de importante en su curso, comunica con claridad a los estudiantes lo que se espera de ellos y se adapta con facilidad a diversos contextos. Esta información se incluye con el programa de la materia y los estudiantes definen sus estrategias de estudio con base en ella (Robins, 2001). La tecnología computacional ofrece la posibilidad de simplificar el proceso de evaluación, a través de la gestión en línea de documentos generados con las tareas fuera de clase y organizados en expedientes, o portafolios electrónicos, generados y operados con el uso de aplicaciones web de libre acceso, como Google docs, en http://docs.google.com.

Se eligen las aplicaciones de Google Docs por considerarla una herramienta importante en apoyo para la educación. El Centre for Learning \& Performance Technologies realizó un "diccionario de herramientas para el aprendizaje" donde aparecen 2,400 herramientas. En su elaboración participaron expertos en aprendizaje y profesionales de educación. Como se aprecia en la Tabla 1, la preferencia de Google Docs ocupa el número 12, lo que implica que tiene un lugar destacado en este ranking. Esta herramienta posee, además, características importantes como la adaptabilidad, mobilidad y apoyo al aprendizaje cooperativo.

Existen diversos sistemas para evaluar el aprendizaje en la educación superior. Uno de los más comunes es el que se basa en criterios para asignar calificaciones a los diversos aspectos que se toman como evidencias del aprendizaje logrado. A partir de los objetivos que se pretenden alcanzar, se diseñan las actividades de evaluación para medir el nivel de logro en cada uno de ellos y su importancia relativa. 
Tabla 1: Herramientas preferidas para el aprendizaje, según el "directorio" de 2008 elaborado por el Centre for Learning \& Performance Technologies http://c41pt.co.uk/recommended/top100.html Tomado de: Coll y Monereo (2008).

\begin{tabular}{|l|l|l|l|l|}
\hline $\begin{array}{l}\text { Ranking } \\
2008\end{array}$ & $\begin{array}{l}\text { Nombre de la } \\
\text { herramienta }\end{array}$ & Descripción & $\begin{array}{l}\text { Software Libre } \\
\text { propietario }\end{array}$ & $\begin{array}{l}\text { Descargable } \\
\text { En línea }\end{array}$ \\
\hline 1. & Del.icio.us & Folksonomía de páginas web & Libre & En línea \\
\hline 2. & Firefox & Navegador web & Libre & Descargable \\
\hline 3. & Google Reader & Lector de feed & Libre & En línea \\
\hline 4. & Skype & $\begin{array}{l}\text { Mensajería instantánea. Comunicación } \\
\text { VolP }\end{array}$ & Libre & Descargable \\
\hline 5. & Google Search & Buscador & Libre & En línea \\
\hline 6. & Wordpress & Herramienta de blogs & Libre & $\begin{array}{l}\text { Descargable } \\
\text { y en línea }\end{array}$ \\
\hline 7. & PowerPoint & Herramienta de presentaciones & propietario & Descargable \\
\hline 8. & Blogger & Herramienta de blogs & Libre & En línea \\
\hline 9. & Audacity & Editor de sonido y grabador & Libre & Descargable \\
\hline 10. & Wikipedia & Enciclopedia en línea & Libre & En línea \\
\hline 11. & Gmail & Programa de correo web & Libre & En línea \\
\hline 12 & Google Docs & $\begin{array}{l}\text { Sitio para alojar, procesar y compartir } \\
\text { documentos }\end{array}$ & Libre & En línea \\
\hline
\end{tabular}

La subjetividad es un aspecto ineludible al evaluar el aprendizaje, de ahí la necesidad de diversificar las oportunidades de evaluación para incrementar la validez de la calificación final, "en general, cuanto mayor sea el número de los elementos utilizados para determinar la calificación, mayor será su validez y confiabilidad" (UoM, 2011). La calificación final se obtiene con la aplicación de la conocida fórmula para determinar promedios ponderados (Weiers, 2006):

$$
\bar{X}_{w}=\frac{\sum w_{i} x_{i}}{\sum w_{i}}
$$

La ecuación indica que la calificación final, $\bar{X}_{w}$, se obtiene al dividir la suma de los productos de las calificaciones de cada uno de los elementos considerados, $X_{i}$, por su ponderación o nivel de importancia correspondiente, $w_{i}$, entre la suma de estas ponderaciones. Cuando estas últimas se expresan en porcentaje, $p_{i}$, la expresión se simplifica a su forma más conocida:

$\bar{x}_{w}=\sum p_{i} x_{i}$

\section{MODELO PROPUESTO}

Las fases y elementos del modelo se presentan en la figura 1. Inicia con la definición de las actividades de aprendizaje por parte del docente (paso 1), quien las registra en línea (paso 2) para que sean consultadas por los estudiantes (paso 3), los cuales generan los documentos y los registran en su expediente en línea para ser considerados como evidencias (paso 4), donde los revisa y evalúa el docente (paso 5), el cual realimenta de manera individual y grupal a los estudiantes, con las observaciones pertinentes (paso 6). 


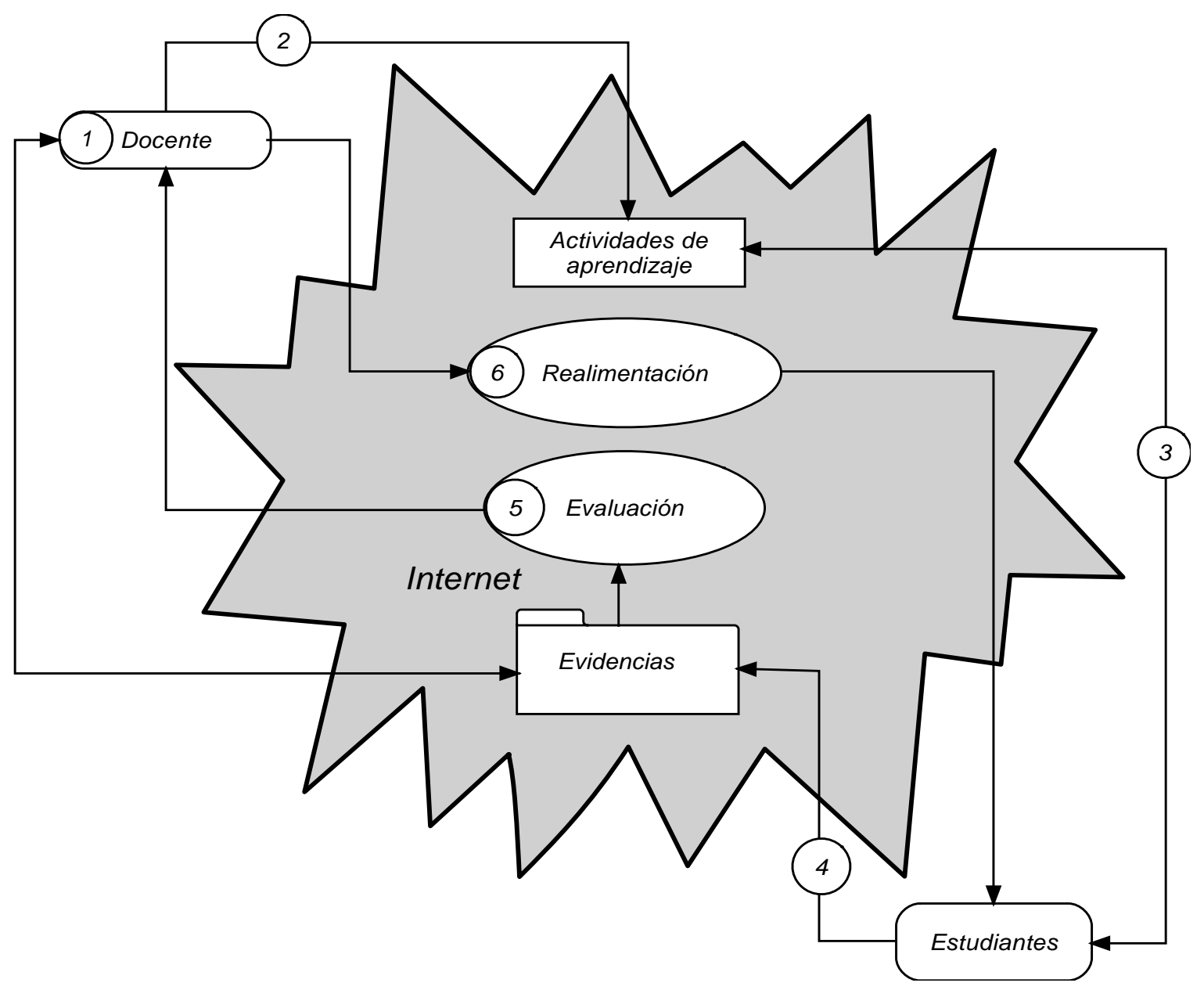

Fig. 1: Modelo de gestión en línea de evidencias de aprendizaje

Este sistema para determinar calificaciones ofrece la posibilidad de incluir los aspectos que se consideren necesarios, así como la libertad para asignar el nivel de relevancia de cada uno de ellos en la calificación final. La disponibilidad de herramientas informáticas, cada vez más accesibles, simplifica la realización de los cálculos. Un incremento en el número de evaluaciones multiplica la carga de trabajo para el docente, de ahí la necesidad de apoyo en sistemas computacionales que simplifiquen esta labor. La web ofrece diversas herramientas para gestionar documentos producidos como tareas fuera de clase. El modelo que aquí se describe toma como base las aplicaciones de libre acceso del sitio Google docs (www.google.com.mx).

El modelo consta de una base de datos con la información general de los estudiantes, en la cual éstos pueden consultar los resultados de sus evaluaciones, así como de carpetas personales donde coleccionan los productos de sus tareas, creados con el uso de aplicaciones proporcionadas por el sitio. Las carpetas son creadas por los propios estudiantes y se identifican con el nombre propio de cada uno de ellos; en ellas el profesor anota sus comentarios en los mismos documentos que revisa. Google docs ofrece diversos servicios de apoyo para crear, conservar y compartir documentos en forma controlada. El uso de sus herramientas no requiere de preparación especial en sistemas computacionales por parte de los usuarios, un curso básico de corta duración puede ser suficiente para utilizarlas de forma adecuada, razón por la cual se selecciona para poner en práctica este modelo.

La principal ventaja del modelo propuesto es la eliminación de la necesidad de manipular y transferir documentos, físicos o electrónicos, debido a que éstos se generan y registran en la cuenta del estudiante, debidamente ordenados en su carpeta correspondiente, a la cual accede el profesor a través de internet. En contrapartida, su principal limitación es la inseguridad en cuanto a la permanencia del servicio. 


\section{MÉTODO}

En el curso de "Estadística I" que uno de los autores imparte en el Centro Universitario de Ciencias Económico Administrativas de la Universidad de Guadalajara (UdeG), se propuso a los estudiantes utilizar este modelo para gestionar los productos de las tareas realizadas fuera del aula, una vez que se explicaron las principales características del mismo. En el estudio participaron 192 estudiantes, 89 mujeres (M) y 103 hombres $(\mathrm{H})$, de diversas carreras, durante los ciclos semestrales 2010B y 2011A:

\section{Carrera:}

Licenciatura en Administración

Licenciatura en Administración Financiera y Sistemas

Licenciatura en Administración Gubernamental y Políticas Públicas Locales

Licenciatura en Economía

Licenciatura en Mercadotecnia

Licenciatura en Negocios Internacionales

Licenciatura en Recursos Humanos

Licenciatura en Sistemas de Información

Licenciatura en Turismo

Licenciatura en Urbanística y Medio Ambiente

Totales:

$\begin{array}{rrr}\mathrm{M} & \mathrm{H} & \text { Total } \\ 17 & 32 & 49 \\ 5 & 6 & 11 \\ 0 & 6 & 6 \\ 3 & 6 & 9 \\ 24 & 14 & 38 \\ 10 & 11 & 21 \\ 8 & 8 & 16 \\ 1 & 10 & 11 \\ 21 & 8 & 29 \\ 0 & 2 & 2 \\ 89 & 103 & 192\end{array}$

Se creó un expediente para los documentos relacionados con el curso, al cual tuvieron acceso todos los estudiantes registrados, sin la posibilidad de realizar modificaciones a los documentos ahí consignados, derecho reservado al profesor, quien genera y administra los contenidos de este expediente. Estos documentos son, entre otros, los siguientes:

1. Programa de la materia

2. Guía para la gestión de tareas

3. Fórmulas estadísticas

4. Copias de las presentaciones proyectadas en clase

5. Lista de asistencias con evaluaciones

\section{Avisos varios.}

Los estudiantes crearon, por su parte su propia carpeta o expediente digital, para conservar los documentos producidos por ellos mismos, algunos de ellos con el apoyo de otros estudiantes. Las comunicaciones personales profesor-estudiante y estudiante-profesor se realizaron a través del servicio de correo del mismo sitio gmail, http://mail.google.com.

Se aplicó una encuesta al final del curso a dos grupos de 46 alumnos cada uno; una de las preguntas de esta encuesta fue ¿cuál es su opinión sobre el uso de las computadoras en la educación superior? El cuestionario se aplicó con el módulo para formularios de Google Docs. Se recibieron 82 respuestas, $89 \%$ del total de estudiantes, 43 hombres y 39 mujeres. A excepción de los dos estudiantes de la carrera de Urbanística y Medio Ambiente, todos habían tomado al menos un curso introductorio sobre informática básica.

\section{RESULTADOS}

De los 82 estudiantes que respondieron la encuesta, 37 (45\%) consideran que el uso de las computadoras ayudan al aprendizaje, 32 (39\%) indican que se trata de una herramienta necesaria en la educación superior, 2 de ellos reconocen riesgos por la atracción que ejercen las redes sociales, que pueden distraer la atención y estorbar al aprendizaje. Sólo uno considera más efectivo al método tradicional y otro más percibe que la tecnología restringe la participación de los estudiantes. Otros perciben algunas limitaciones prácticas o la necesidad de más capacitación para utilizarlas con mayor efectividad. A continuación se muestran algunas de las respuestas que se consideran más representativas de la percepción de los estudiantes sobre el uso de la tecnología informática en la educación superior: 
Tabla 2: Respuestas de algunos estudiantes y comentarios a las mismas

\begin{tabular}{|c|c|c|}
\hline Participante* & Respuesta & Comentario \\
\hline EHNIN1 & $\begin{array}{l}\text { "Pienso que benefician mucho tanto al alumno como } \\
\text { al maestro, ya que es una forma de adaptarte más a } \\
\text { realizar trabajos en nuevos sistemas de cómputo, } \\
\text { también beneficia al medio ambiente ya que reduce el } \\
\text { uso de papel". }\end{array}$ & $\begin{array}{l}\text { Percibe beneficios inmediatos y } \\
\text { de largo plazo. }\end{array}$ \\
\hline EMTURA9 & $\begin{array}{l}\text { "Es una forma más sencilla y cómoda para dar la } \\
\text { clase, además de hacerla dinámica, facilita el } \\
\text { aprendizaje porque mantiene la atención del } \\
\text { estudiante". }\end{array}$ & $\begin{array}{l}\text { Identifica la comodidad, } \\
\text { sencillez y dinamismo para } \\
\text { impartir clases, además de que } \\
\text { capta y mantiene la atención de } \\
\text { los estudiantes. }\end{array}$ \\
\hline EMAFS2 & $\begin{array}{l}\text { "Facilita el trabajo [...]. La forma en la que afecta es el } \\
\text { riego de pérdida de información mediante virus u otras } \\
\text { situaciones, por ello es importante siempre tener } \\
\text { respaldada toda información". }\end{array}$ & Identifica un riesgo \\
\hline EHTURA1 & $\begin{array}{l}\text { “...me parece que es una manera cómoda para } \\
\text { entregar los trabajos, pero en mi opinión se vuelve } \\
\text { algo tedioso y me parece mejor para el aprendizaje de } \\
\text { materias como estadística y matemáticas el método } \\
\text { práctico" [tradicional]. }\end{array}$ & $\begin{array}{l}\text { Percibe limitaciones y } \\
\text { desventajas }\end{array}$ \\
\hline EMMER13 & $\begin{array}{l}\text { "Creo que es importante su uso, y que además se } \\
\text { debe utilizar más debido a que es una herramienta } \\
\text { que facilita el resolver problemas, que está bien que } \\
\text { podamos conocerla. Sin embargo, también es } \\
\text { importante que nos enseñen la parte manual: cómo } \\
\text { razonar, cómo resolverlo nosotros mismos porque lo } \\
\text { entendimos". }\end{array}$ & $\begin{array}{l}\text { Percibe la tecnología como un } \\
\text { elemento complementario para } \\
\text { la enseñanza. }\end{array}$ \\
\hline EHRHU1 & $\begin{array}{l}\text { "Son de suma importancia, ya que lo jóvenes tienen } \\
\text { que aprender a usar las nuevas tecnologías ya que en } \\
\text { lo laboral en cualquier empresa te van a pedir el uso } \\
\text { de computadoras y de nuevas tecnologías". }\end{array}$ & $\begin{array}{l}\text { Percibe la necesidad de conocer } \\
\text { mejor esta tecnología }\end{array}$ \\
\hline \multicolumn{3}{|c|}{$\begin{array}{l}\text { EHNIN1: Estudiante hombre de la licenciatura de sistemas de información número } 1 . \\
\text { EMTURA9: Estudiante mujer de la licenciatura de turismo número } 9 . \\
\text { EMAFS2: Estudiante mujer de la licenciatura administración en sistemas financieros número } 2 . \\
\text { EHTURA1: Estudiante hombre de la licenciatura de turismo número } 1 . \\
\text { EMMER13: Estudiante mujer de la licenciatura de mercadotecnia número } 13 . \\
\text { EHRHU1: Estudiante hombre de la licenciatura de recursos humanos número } 1 .\end{array}$} \\
\hline
\end{tabular}

\section{CONCLUSIONES}

La adopción de la tecnología informática en la educación superior presenta diversos obstáculos, desde la disponibilidad de los recursos necesarios, hasta la disposición por parte de docentes y estudiantes para aprender a utilizarlos de forma adecuada. La evolución que presenta esta tecnología hace cada vez más accesible su disponibilidad en las instituciones educativas, lo que acrecienta el interés por desarrollar métodos para integrarla de manera efectiva a las actividades educativas.

Los estudiantes perciben beneficios y algunos riesgos, pero en general consideran útil y necesario su adopción en la educación superior, porque es una forma de recibir retroalimentación a sus actividades de evaluación y es una evidencia que perdura en el sitio web.

El modelo para gestionar en línea evidencias de aprendizaje resultó exitoso para el proceso enseñanza aprendizaje al apoyarse en algunas herramientas web de libre acceso para las actividades de evaluación del desempeño académico de los estudiantes no sólo simplifica el proceso, sino que fortalece el desarrollo de competencias que los estudiantes perciben como 
necesarias para su vida profesional. Dicho modelo logra auxiliar los cursos que la UdeG ofrece de forma presencial como a la modalidad mixta.

Entre las herramientas disponibles, para este trabajo se seleccionó a Google docs por la relativa facilidad para acceder y utilizar sus aplicaciones. Sus herramientas simplifican el proceso de gestión de documentos, desde la generación, hasta la organización, almacenamiento y control de acceso a los mismos.

\section{REFERENCIAS}

Bates, A. W., Cómo gestionar el cambio tecnológico, Gedisa, España (2001).

Barberá E., Bautista, G., Espasa, A. y Guasch, T. Portfolio electrónico: desarrollo de competencias profesionales en la red. En Revista de Universidad y Sociedad del Conocimiento, 3(2), 55-66 (2006)

Coll, C., y Monereo, C. Psicología de la educación virtual. Morata, Madrid (2008)

Ginns, P. y Ellis, R., Quality in blended learning: Exploring relationships between on-line and faceto face teaching and learning. En Internet and Higher Education 10. 53-64 (2007)

Gipps, C., Beyond Testing: Towards a theory of Educational Assessment. Falmer Press, London (1994)

James, R., McInnis, C., y Devlin, M., Assessing Learning in Australian Universities. Centre for the Study of Higher Education. http://www.cshe.unimelb.edu.au/assessinglearning/ Recuperado el 8 de noviembre de 2008.

Laurillard, D., E-Learning in Higher Education. En Changing Higher Education: The development of learning and teaching. Edited by Ashwin, P. Routledge, London (2006)

Marzano, R., Classroom Assessment and Grading that Work. Association for Supervision and Curriculum Development (ASCD), Alexandria, Virginia, U.S.A. (2006)

McDonald, E., Getting Out of the Assessment Rut. Inspiring Teachers Publishing, Inc. http://inspiringteachers.com/classroom_resources/articles/assessment/out_of_the_rut.html Recuperado el 8 de marzo de 2011.

Norton, L., Using assessment to promote quality learning in higher education. En Learning, Teaching and Assessing in Higher Education: Developing Reflective Practice. Campbell, A., y Norton, L. (Editores), Exeter, EX! 1NX, Devon, U.K. (2007)

Palfrey, J. y Gasser, U., Opening Universities in a Digital Era. The New England Journal of Higher Education, 23(1), p. 22 (2008).

Pellegrino, J., Chudowsky, N., y Glaser, R., Knowing what Students know: The Science and Design of Education Assessment, National Academy Press, Washington, D.C. (2001).

UoM, University of Minnesota, Grading Systems. Online Teaching Resources. http://www1.umn.edu/ ohr/teachlearn/resources/grading/index.html, Recuperado el 1 de marzo de 2011.

Weiers, R., Introducción a la estadística para negocios. Quinta edición, International Thomson Editores. México, D.F. (2006) 